\title{
RESISTANCE TRAINING EXERCISE EFFECTS ON MUSCULAR STRENGTH, ENDURANCE, AND FATS OF LOWER LIMBS IN ADULTS; IT'S FINANCIAL IMPLICATIONS
}

\author{
Noor Muhammad Marwat ${ }^{1 *}$, Aziz Javed ${ }^{2}$, Pir Muhammad Abdul Aziz Shah ${ }^{3}$, Sher Zaman ${ }^{4}$, Muhammad Aslam \\ $\mathrm{Khan}^{5}$ \\ ${ }^{1 *}$ Lecturer, Department of Sports Sciences and Physical Education, Gomal University D.I.Khan, Pakistan; ${ }^{2}$ Assistant \\ Professor, Institute of Business Administration, Gomal University, D.I.Khan, Pakistan; ${ }^{3} \mathrm{Ph}$.D. Scholar, University of \\ Sindh Jamshoro, Pakistan; ${ }^{4}$ Lecturer, Department of Commerce Gomal University D.I.Khan, Pakistan; ${ }^{5}$ Assistant \\ Professor, Department of Pakistan Studies, Gomal University, D.I.Khan, Pakistan. \\ Email: ${ }^{1 *}$ marwatnoor@yahoo.com, ${ }^{2}$ azizjaved73@gmail.com, ${ }^{3}$ peershah4880@gmail.com, ${ }^{4}$ shkhan@ gu.edu.pk, \\ 5draslamkhan@gu.edu.pk
}

Article History: Received on $2^{\text {nd }}$ July 2021, Revised on $25^{\text {th }}$ July 2021, Published on $27^{\text {th }}$ July 2021

\begin{abstract}
Purpose: Primary aim of the research study was to develop awareness among the participants about the benefits of exercise and its financial benefits.

Methodology: Adults had to perform exercises on Unstable Surfaces (US), Stable Surface (SS) and Machine Based (MB). The protocol of exercises was divided into three categories with 30 participants in each group i.e., Machine Based Surface Resistance Training (M-SRT), Machine Based Unstable Surface Resistance Training (M-URT), and Free Weight Unstable Surfaces Resistance Training (F-URT). Equipment used for tests was imported. Cost of financial expenditure and effects of resistance training exercise protocol on the male adults' lower limbs muscular power, body fats, and strength were compared. Leg extension strength, leg press, muscle fuel, and body fats were checked on the baseline and after a seven-week resistance training program in the adults and budget, effects took away from the parents.

Main Findings: The subjects' results in maximal resistance training of squad movement were significantly higher in MSRT and M-URT as compared to F-URT. The average seven-week regular resistance training exercise protocol program in adults with proper supervision will minimize the risk of injury and develop the strength and endurance in the lower limbs of adults up to maximal and that the composition of the body fats was observed lesser. There are training and exercise centres in Dera Ismail Khan city and adults can easily manage to become members of those clubs with nominal registration fees. Fitness and wellbeing are directly associated with the financial benefits of exercise and training. It was observed that the student adults can easily manage the resistance training program for their fitness by curtailing their pocket money.
\end{abstract}

The implication of the Study: The findings of the research study have vital impacts on the daily life routine life of adults. Adults can easily manage to spare some time from leisure and money to keep themselves fit and healthy. The nation needs healthy adults to serve the best and build a nation. The results of analyzed data will help the parents and teachers to motivate their children to participate in the activities of fitness centres and acquaint the adults with financial behaviour change theory. Proper counselling of the experts will be fruitful in light of the recommendations of the study.

Novelty: For the first time such a study is going to be conducted in the field of sports and finance. This is primary research in the homeland to highlight the behavioural economics capitalization in sports and training. The financial impacts of training and exercise on the fitness of youth have been studied in length. Future researchers would be benefited from the results of the study and they will be able to include the purchase of basic equipment for exercise and training in the institute budget.

Keywords: Resistance Training, Exercise, Muscular Strength, Endurance, Limbs, Financial Implications.

\section{INTRODUCTION}

In the human body legs are pillars of the body and one must concentrate on their proper strength and training. This must be set as a priority goal is to keep the lower extremity fit and ample power. Resistance training programs and exercises improve the muscular force production capacity in adults. A Regularly scheduled training program of exercise protocol is essential for the rapid reaction for controlling body posture. It also improves the static and dynamic balance in adults. Exercises may be aerobic, muscular strength, balancing, walking, and jogging (Lopes CR Aoki MS Crisp AH, 2017). Resistance training (RT) for adults with a low, moderate, and higher level of loads have positive effects on the power and strength of lower limbs as well as muscular hypertrophy. In a supervised resistance training program for lower extremities, the products of higher and moderate levels of loads were superior as compared to the lower loads training program. It was also analyzed that in untrained adults more muscular hypertrophy was exhibited (Schoenfeld BJ, 2016). In another research study, it was found that strength and resistance training of adults is key to flexibility, mobility, athletic ability, enhanced performance, and minimizing risks of injuries. Strength and resistance training programs need not much more formalities and they can be improved in anybody. The modern age technology and pace of life have evolved tremendously but unfortunately, the human body has been the same since its creation. We need not fancy and 
fantasies with advanced equipment but need just sparing time for exercise and training to correct body weight. It will enable us to remain fit, flexible, and have proper control over muscular movements (Jenkins NDM Miramonti AA Hill EC, 2017). The results of research where discussing muscular strength and muscular endurance have elaborated that muscular strength-related exercises in adults improve the ability to move accurately and lift objects properly. The training program enhances the time to resist the external stimulus and lift/sustain more weight for more time. The program of exercises may include; lifting/holding heavier weights for fewer repetitions, cycling, climbing hills and running, etc. (Lesinski M, 2015). There are vital benefits of financial incentives on athletes' involvement in sports and

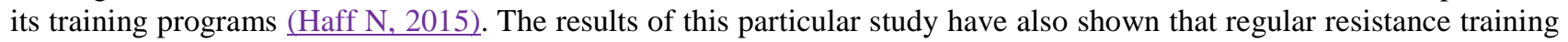
program for adults makes the muscles more rigid than normal. The participants enjoy the benefits of a strong body with lean muscle mass. The bones density is increased with the improvement in the range of motions. Resistance training programs improve the brain health of adults and cognitive functions. The energy level of the resistance training program participants is enhanced and body weight is reasonably reduced. Seven-week controlled resistance training program for the strength of lower extremities in adults when properly supervised has a significant effect on the strength, power, and obesity control (B. Horsak, 2015). Finance is no problem if the participant is fit and free of worries, these were results findings of research conducted in the USA in various offices on employees (Capps KH, 2008). Individual and team sports events extend a lot of financial assistance to the participants (Patel-M, 2016). The exercise and resistance training program of 10 -week in adults had $95 \%$ and $60 \%$ improved the strength and power of the lower limbs of adults respectively in players and starters (Muñoz-Martínez F.A., 2017). Sports and training programs for the fitness of adults are enhanced due to behavioural economics principles with accurate capitalizing decision making (Adams J, 2014). The contribution of financial contribution on the awareness levels of behavioural economics and behaviour change theory has paramount positive effects on adults' exercise and sports training programs (Michie. S, 2010). Results of earlier studies of (Mitchell MS, 2013, Molema CC, 2016) have reported that the financial benefits of exercise and sports training programs are directly associated with the fitness and well-being of participants. The power of the leg extension was improved up to $25.3 \%$ and leg muscle strength improved up to $75.5 \%$, in male adults participating in an observational study of the resistance training program (Pollock M., 2016). The resistance training program consisting of low frequency for one day per week and with high frequency at least three days per week has brought significant changes and betterment in the lower limbs' strength, power, and explosive power. The participants have reported reasonable weight loss after the resistance training exercise program (Lopes CR Aoki MS Crisp AH, 2017, and Pollock M., 2016).

The main theme of the study is to provide guidelines for the selection of resistance training loads for the development of muscular strength and endurance of the lower limbs and controlling weight in adults.

\section{Objectives of the Study}

1. To find out the impact of resistance training on the lower limbs of adults.

2. To check the endurance level of adults after resistance training in adults.

3. To evaluate the financial impact of training on the participants budgeting.

\section{Hypotheses}

Ho 1: There is no sign of resistance training in adults.

Ho 2: There is no significant effect of resistance training on the endurance level of adults.

Ho 3: There is no significant impact of resistance training on the budget of the participants.

\section{RESEARCH METHOD}

The researcher got proper approval from the research ethnic committee of the Department of Sport Sciences and Physical Education, Gomal University, Dera Ismail Khan, Khyber Pakhtunkhwa, Pakistan. Subjects were 90 male adult students from Government Degree Colleges of Dera Ismail Khan City, with the age group of 15-19 years. All the adults were healthy and willing to participate in the resistance training program. The resistance training program span was seven-week. The training schedule was divided into three categories low, moderate, and high loads for a participant. For the low load category the repetition maximum was $>15$, for moderate it was $9-15$ RM, and for high loads, it was $<8$ Repetition Maximum. The adults were also split down into three groups i.e., first Machine Based Resistance Training group (M-SRT), the second Machine Based Unstable Surface Resistance Training (M-URT), and the third Free Weight Unstable Surface Resistance Training (F-URT). All participants of three groups had to participate in the scheduled supervised training program two times per week with a session of one hour. The protocol of exercises included HighIntensity Interval Training, Isokinetic Strengthening, Leg Extension Strength, Chair Rise Test, and Functional Reach Test. The training program span was seven-week for all three groups, training was conducted under the strict supervision of experts in the field. For all the adults in each attempt, the subjects were required to perform as many continuous repetitions as possible and thus trial was terminated when the subject was unable to maintain a symmetrical controlled steady rhythm. The completed repetitions were counted out after each repetition by one of two attendants assisting the subjects. Testing was terminated for those subjects who could not reach at ' 6 ' repetitions in one trial. To move onto the 
next higher load setting subjects needed to perform most elevated in the cadre repetitions at least in three trials in the previous load. The data were regularly collected and tabulated.

\section{Participants}

Subjects were 90 male adult students from Government Degree Colleges of Dera Ismail Khan City, with the age group of 15-19 years. All the adults were healthy and willing to participate in the resistance training program.

\section{Instrument}

The resistance training program span was seven-week. The training schedule was divided into three categories low, moderate, and high loads for the participants. For the low load category the repetition maximum was $>15$, for moderate it was 9-15 RM, and for high loads, it was <8 Repetition Maximum. Adults were also split down into three groups i.e., first Machine Based Resistance Training group (M-SRT), second Machine Based Unstable Surface Resistance Training (M-URT), and third Free Weight Unstable Surface Resistance Training (F-URT). All the participants of three groups had to participate in the scheduled supervised training program two times per week with a session of one hour. The protocol of exercises included High-Intensity Interval Training, Isokinetic Strengthening, Leg Extension Strength, Chair Rise Test, and Functional Reach Test.

\section{Reliability}

The reliability of the tests applied was checked and it was well within the limits.

\section{Data Analysis}

Table 1: F-URT (Free Weight Unstable Surface Resistance Training)

\begin{tabular}{lllll}
\hline S.No. & Protocol of Exercise & Base Line & After Resistance Training & Percentage \\
\hline 1. & $\begin{array}{l}\text { Knee Extensor(KE) } \\
1-\mathrm{RM}(\mathrm{Kg})\end{array}$ & $32 \pm 4$ & $43 \pm 4$ & $34.375 \%$ \\
\hline 2. & $\begin{array}{l}\text { Leg Press } \\
(\mathrm{Kg})\end{array}$ & $110 \pm 7$ & $121 \pm 7$ & $10.00 \%$ \\
\hline 3. & $\begin{array}{l}\text { Power } \\
(\text { watts })\end{array}$ & $376 \pm 20.50$ & $438 \pm 19.01$ & $16.489 \%$ \\
\hline 4. & $\begin{array}{l}\text { Body Fats } \\
(\%)\end{array}$ & $33.24 \pm 1.02$ & $33.01 \pm 0.92$ & $-0.691 \%$ \\
\hline
\end{tabular}

Results of the above table show that the strength of the knee extensor was improved up to $34.75 \%$ in the adults, leg press to $10 \%$, and power to $16.489 \%$ in the adults who participated in the resistance training program. It has also been found out that $0.691 \%$ of fats were burnt from the participants' bodies due to resistance training.

Table 2: M-URT (Machine Based Unstable Resistance Training)

\begin{tabular}{lllll}
\hline S.No. & Protocol of Exercise & Base Line & After Resistance Training & Percentage \\
\hline 1. & $\begin{array}{l}\text { Knee Extensor(KE) } \\
1-\mathrm{RM}(\mathrm{Kg})\end{array}$ & $32 \pm 4$ & $59 \pm 4.6$ & $84.375 \%$ \\
\hline 2. & $\begin{array}{l}\text { Leg Press } \\
(\mathrm{Kg})\end{array}$ & $110 \pm 7$ & $150 \pm 8$ & $36.363 \%$ \\
\hline 3. & $\begin{array}{l}\text { Power } \\
(\text { watts })\end{array}$ & $376 \pm 20.50$ & $473 \pm 25.43$ & $25.797 \%$ \\
\hline 4. & $\begin{array}{l}\text { Body Fats } \\
(\%)\end{array}$ & $33.24 \pm 1.02$ & $25 \pm 1.00$ & $-24.789 \%$ \\
\hline
\end{tabular}

In the above table, the results of Machine Based Unstable Resistance Training exercise protocol effects on the male adult's lower extremities have been shown. It is clear from the figures that knee extension was improved to $84.375 \%$, leg press $36.363 \%$, and power $25.797 \%$. The results also depict that $24.789 \%$ lower lipid level was seen after the resistance training program in adults.

Table 3: M-SRT (Machine Based Surface Resistance Training)

\begin{tabular}{lllll}
\hline S.No. & Protocol of Exercise & Base Line & After Resistance Training & Percentage \\
\hline 1. & $\begin{array}{l}\text { Knee Extensor(KE) } \\
1-\mathrm{RM}(\mathrm{Kg})\end{array}$ & $32 \pm 4$ & $58 \pm 6$ & $81.25 \%$ \\
\hline 2. & $\begin{array}{l}\text { Leg Press } \\
(\mathrm{Kg})\end{array}$ & $110 \pm 7$ & $147 \pm 8.09$ & $33.636 \%$ \\
\hline 3. & $\begin{array}{l}\text { Power } \\
\text { (watts) }\end{array}$ & $376 \pm 20.50$ & $467 \pm 22.00$ & $24.202 \%$ \\
\hline
\end{tabular}




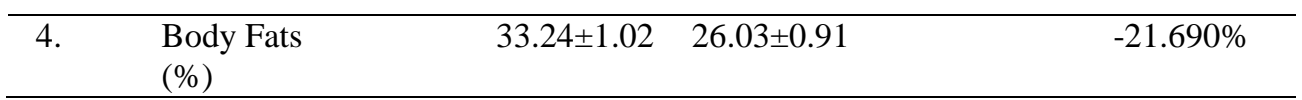

Results of table No: 3 show that $81.25 \%$ knee extension, $33.636 \%$ leg press, and $24.202 \%$ power in the lower limbs of trainee adults were enhanced. It is also clear from the results that $21.690 \%$ less fats were observed from the baseline after the resistance training program in the male adults. These results are supported by the study out in 2016, by Morton $\underline{\mathrm{RW}}$.

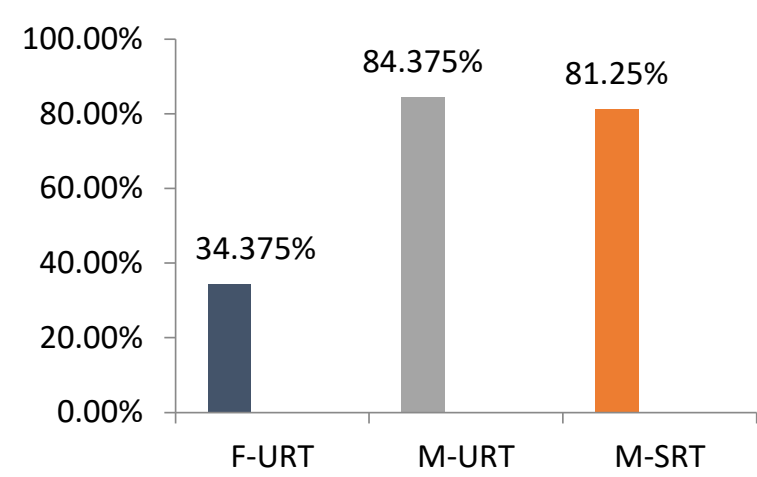

Figure 1: Group Wise Graphical

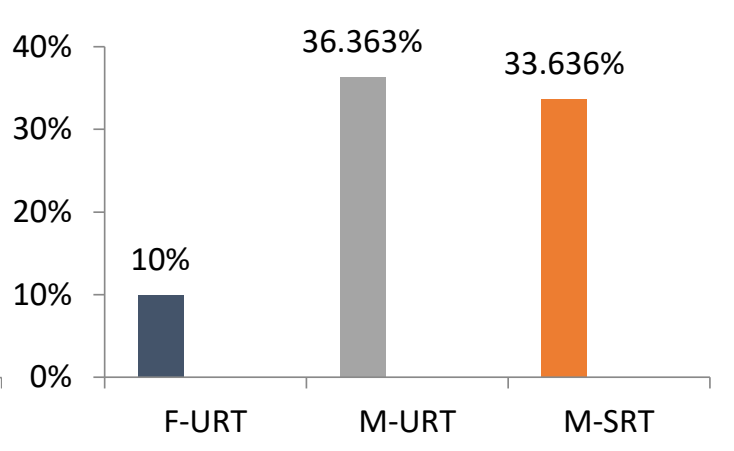

Figure 2: Group Wise Graphical

\section{Comparison of Knee Extensor 1-RM (Kg). Comparison of Leg Press (Kg).}

The above figure 1 shows the results of the sample tested for Knee Extensor strength and Leg Press after the seven-week resistance training program. The change in knee extension of the M-URT group led in percentage is greater than the remaining groups. In figure 2 it is also prominent that Leg Press results from percentage was higher in the same group as compared to other groups. In an earlier study such like comparison was made, matching the results of our study (Rucker G, 2017).

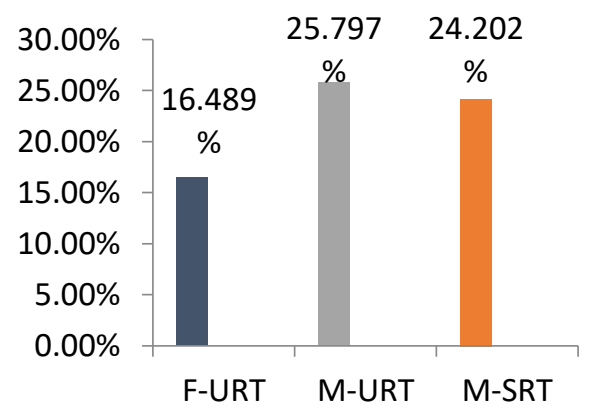

Figure 3: Group Wise

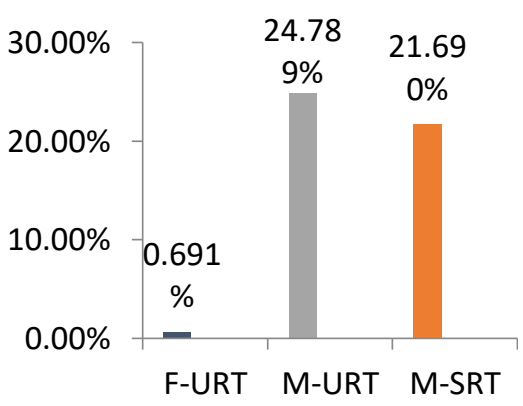

Figure 4: Group Wise

\section{Graphical Comparison of Power (Watts.) Graphical Comparison of Body Fat $(\%)$.}

Results of Figures $3 \& 4$ as also the same here in both results the percentage of the M-URT program is also superior to F-URT and M-SRT.

\section{DISCUSSION}

Results of the exercise protocol of the resistance training program of seven-week for the three groups were statically analyzed and it has been shown in the below tables. The lower extremities resistance training exercise protocol program on even and uneven surfaces undoubtedly improved the strength, leg press, and power of the limbs of participants, which were at par with the results of Cholewa JM Rossi (2018). Schoenfeld BJ (2017) results are at par with the findings of the study that fats were also reasonably burnt, participants felt more fit, energetic, and lighter after the seven-week regular resistance training program. In 2019, a research study was carried out by Sterne JAC Savovic and indicated the results favouring a comparison of the above three table findings. Where it has been elaborated that Free Weight Unstable Surface Resistance Training (F-URT) program results were significantly lower as compared to Machine Based Unstable Surface Resistance Training Program (M-URT) and Machine Based Surface Resistance Training Program (M-SRT)

\section{CONCLUSION}

Results of the research have shown that both objectives have been achieved. Resistance training has a lot of positive effects on the fitness level of participants. No financial impact of resistance training and exercise programs was observed. All the participants of the research study would be best motivators for the others who ignore their fitness and well-being due to speculated financial impact. 


\section{LIMITATIONS}

Researchers having in mind the financial constraints and Covid-19 prevailing situations restricted the study only to the adults of Lakki Marwat, KP, Pakistan.

\section{AUTHORS CONTRIBUTION}

Noor Muhammad Marwat ${ }^{1}$, Aziz Javed ${ }^{2}$, Muhammad Aslam Khan ${ }^{3}$, Sher Zaman ${ }^{4}: 1$ - Study design; 2 - Data collection; 3- Statistical analysis; 4 - Manuscript preparation, 5- Financial Management.

\section{REFERENCES}

1. Adams, J., Giles, E.L., McColl, E., Sniehotta, F.F., Carrots. (2014). Sticks and health behaviours: a framework for documenting the complexity of financial incentive interventions to change health behaviours. Health Psychol Rev, 8(3), 286-95. https://doi.org/10.1080/17437199.2013.848410

2. Horsak, B., Artner, D., Baca, A., Pobatschnig, B., Greber-Platzer, S., Nehrer, S., Wondrasch, S. (2015). The effects of a strength and neuromuscular exercise programme for the lower extremity on knee load, pain and function in obese children and adolescents: study protocol for a randomised controlled trial. National library of science. https://doi.org/10.1186/s13063-015-1091-5

3. Capps, K.H., John, B. (2008). Employee health \& productivity management programs: the use of incentives. A survey of major U.S. employers. The results of the Spring 2008 Health, Wellness \& Productivity Programs, Incentives \& ROI Impact Survey. Systematic Reviews.

4. Cholewa, J.M., Rossi, F.E., MacDonald, C, et al. (2018). The effects of moderate- versus high-load resistance training on muscle growth, body composition, and performance in collegiate women. J Strength Cond Res, 32(6), 11-24. https://doi.org/10.1519/JSC.0000000000002048

5. Giles, E.L., Robalino, S., McColl, E., Sniehotta, F.F., Adams, J. (2014). The effectiveness of financial incentives for health behaviour change: systematic review and meta-analysis. PLoS One, 9(3), e90347. https://doi.org/10.1371/journal.pone.0090347

6. Haff, N., Patel, M.S., Lim, R., Zhu, J., Troxel, A.B., Asch, D.A., Volpp, K.G. (2015). The role of behavioral economic incentive design and demographic characteristics in financial incentive-based approaches to changing health behaviors: a meta-analysis. Am J Health Promot, 29(5), 314-23. https://doi.org/10.4278/ajhp.140714LIT-333

7. Jenkins, N.D.M., Miramonti, A.A., Hill, E.C., et al. (2017). Greater neural adaptations following high- vs. lowload resistance training. Front Physiol, 8, 331. https://doi.org/10.3389/fphys.2017.00331

8. Lesinski, M., Hortobágyi, T., Muehlbauer, T., Gollhofer, A., Granacher, U. (2015). Dose-response relationships of balance training in healthy young adults: a systematic review and meta-analysis. Sports Med, 45, 557-576. https://doi.org/10.1007/s40279-014-0284-5

9. Lopes, C.R. Aoki, M.S., Crisp, A.H., et al. (2017). The effect of different resistance training load schemes on strength and body composition in trained men. J Hum Kinet, 58, 177-86. https://doi.org/10.1515/hukin-2017$\underline{0081}$

10. Michie, S., Prestwich, A. (2010). Are interventions theory-based? Development of a theory coding scheme. Health Psychol, 29(1), 1. https://doi.org/10.1037/a0016939

11. Mitchell, M.S., Goodman, J.M., Alter, D.A., John, L.K., Oh, P.I., Pakosh, M.T., Faulkner, G.E. (2013). Financial incentives for exercise adherence in adults: systematic review and meta-analysis. Am J Prev Med, 45(5), 658-667. https://doi.org/10.1016/j.amepre.2013.06.017

12. Molema, C.C., Wendel-Vos, G.W., Puijk, L., Jensen, J.D., Schuit, A.J., de Wit, G.A. (2016). A systematic review of financial incentives given in the healthcare setting; do they effectively improve physical activity levels? BMC Sports Sci Med Rehabil, 8(1), 15. https://doi.org/10.1186/s13102-016-0041-1

13. Morton, R.W., Oikawa, S.Y., Wavell, C.G., et al. (2016). Neither load nor systemic hormones determine resistance training-mediated hypertrophy or strength gains in resistance-trained young men. J Appl Physiol, 121(1), 129-38. https://doi.org/10.1152/japplphysiol.00154.2016

14. Muñoz-Martínez, F.A., Rubio-Arias, J., Ramos-Campo, D.J., and Alcaraz, P.E. (2017). Effectiveness of resistance circuit-based training for maximum oxygen uptake and upper-body one-repetition maximum improvements: a systematic review and meta-analysis. Sports Med., 47(12), 2553-2568. https://doi.org/10.1007/s40279-017-0773-4

15. Patel, M., Asch, D., Rosin, R., Small, D., Bellamy, S., Eberbach, K., Walters, K., Haff, N., Lee, S., Wesby, L., et al. (2016). Individual versus team-based financial incentives to increase physical activity: a randomized, controlled trial. J Gen Intern Med., 31(7), 746-54. https://doi.org/10.1007/s11606-016-3627-0

16. Pollock, M., Fernandes, R.M., Becker, L.A., Featherstone, R., and Hartling, L. (2016). What guidance is available for researchers conducting overviews of reviews of healthcare interventions? A scoping review and qualitative meta summary. Syst. Rev., 5(1), 190. https://doi.org/10.1186/s13643-016-0367-5

17. Rucker, G., Schwarzer, G., Krahn, U., Konig, J.. (2017). Network meta-analysis using frequentist methods. In R package version 1.2.1.2017. 
18. Schoenfeld, B.J., Contreras, B., Vigotsky, A.D., Peterson, M.. (2016). Differential effects of heavy versus moderate loads on measures of strength and hypertrophy in resistance-trained men. J Sports Sci Med., 15(4), 715-22.

19. Schoenfeld, B.J., Grgic, J., Ogborn, D., Krieger, J.W. (2017). Strength and hypertrophy adaptations between low- vs. high-load resistance training: a systematic review and meta-analysis. J Strength Cond Res., 31(12), 3508-3523. https://doi.org/10.1519/JSC.0000000000002200

20. Sterne, J.A.C., Savović, J., Page, M.J., et al. (2019). RoB 2: a revised tool for assessing risk of bias in randomized trials. BMJ, 366, 14898. https://doi.org/10.1136/bmj.14898 